\title{
Time-variability in the Interstellar Boundary Conditions of the Heliosphere: Effect of the Solar Journey on the Galactic Cosmic Ray Flux at Earth
}

\author{
Priscilla C. Frisch • Hans-Reinhard Mueller
}

Received: date / Accepted: date

\begin{abstract}
During the solar journey through galactic space, variations in the physical properties of the surrounding interstellar medium (ISM) modify the heliosphere and modulate the flux of galactic cosmic rays (GCR) at the surface of the Earth, with consequences for the terrestrial record of cosmogenic radionuclides One phenomenon that needs studying is the effect on cosmogenic isotope production of changing anomalous cosmic ray fluxes at Earth due to variable interstellar ionizations. The possible range of interstellar ram pressures and ionization levels in the low density solar environment generate dramatically different possible heliosphere configurations, with a wide range of particle fluxes of interstellar neutrals, their secondary products, and GCRs arriving at Earth. Simple models of the distribution and densities of ISM in the downwind direction give cloud transition timescales that can be directly compared with cosmogenic radionuclide geologic records. Both the interstellar data and cosmogenic radionuclide data are consistent with two cloud transitions, within the past 10,000 years and a second one 20,000-30,000 years ago, with large and assumption-dependent uncertainties. The geomagnetic timeline derived from cosmic ray fluxes at Earth may require adjustment to account for the disappearance of anomalous cosmic rays when the Sun is immersed in ionized gas.
\end{abstract}

Keywords ISM $\cdot$ Heliosphere $\cdot$ Cosmogenic radionuclides

\section{Introduction}

The Sun has traversed multiple interstellar clouds during it $220 \mathrm{Myr}$ journey around the galactic center, including dense neutral clouds, low density partially ionized interstellar matter (ISM) such as now surrounds the heliosphere, and hot very tenuous plasma. Variations in the cosmic ray fluxes at the surface of the Earth are strongly dependent

Priscilla C. Frisch

University of Chicago, Chicago, IL

E-mail: frisch@oddjob.uchicago.edu

Hans-Reinhard Mueller

Dartmouth College, Hanover, NH

E-mail: hans.mueller@dartmouth.edu 
on the geomagnetic field, the solar magnetic activity cycle, the heliosphere, and the physical properties of the circumheliospheric interstellar material. For example, the modulation of the $1 \mathrm{AU}$ cosmic ray flux by solar flare mass ejections has long been known (Gosling 1964).

The heliosphere acts as a weather vane for the circumheliospheric ISM (CISM), responding to the ionization, magnetic pressure, and dynamic ram pressure Holzer 1989). The cosmic ray component at $1 \mathrm{AU}$ varies with the properties of the heliosphere modulation region. The interpretation of the geological record of cosmogenic isotopes relies on accurate models of the cosmic ray spectra. One factor that is not included in the interpretation of the geological record of cosmogenic isotopes is that the cosmic ray spectrum incident on the Earth consists of two components that behave differently as the Sun travels through space. Galactic cosmic rays dominate at high energies, $>500$ $\mathrm{MeV}$, and are subject to heliospheric modulation as the Sun travels through space. However a second cosmic ray component at lower energies is formed inside of the heliopause from interstellar neutrals that penetrate and are ionized inside of the heliosphere, forming pickup ions. These are subsequently accelerated to form lower-energy anomalous cosmic rays (ACRs) with a composition derived from neutral interstellar atoms in the CISM (Fisk et al. 1974). The local interstellar cosmic ray spectrum that creates the geological radio-isotope record is thus composed of two components that vary differently over time and space, the higher energy galactic cosmic rays (GCRs) that are modulated by a variable heliosphere, and the ACRs that also depend on the density and fractional ionization of the surrounding interstellar cloud.

In this paper we present the overall picture of the ISM characteristics that result from the motion of the Sun and interstellar clouds through space. Observations of interstellar absorption lines towards nearest stars show that spatial variations in velocity, temperature, and ionization of the circumheliospheric ISM create temporal variations in the heliosphere boundary conditions. These then cause temporal variations in the spectrum and fluxes of cosmic rays at Earth. We also draw possible connections between interstellar cloud transitions and the geological radio isotope record.

\section{Contemporary and Paleointerstellar Circumheliospheric ISM}

\subsection{Environment, Dynamics, Structure, and Magnetic Field in Nearby ISM}

Interstellar clouds establish the boundary conditions of the heliosphere, and the heliosphere is the primary modulation region for the galactic cosmic ray flux at Earth. We therefore briefly review the properties of nearby ISM that sets the future and paleointerstellar CISM.

The Sun is traveling through a region with very low mean densities that extends 70-200 pc from the Sun (Figure 1). 11 Stellar winds and supernovae in star formation regions bordering the Local Bubble both sculpted the Local Bubble and contributed to the cosmic ray flux at Earth. Hot low density plasma is widespread in the Local Bubble cavity, $T \sim 10^{6} \mathrm{~K}, n<0.005 \mathrm{~cm}^{-3}$ (Frisch et al. 2009, F09). Within $\sim 15 \mathrm{pc}$, a cluster

\footnotetext{
1 In Fig. 1, the cumulative dust distributions in and beyond the Local Bubble boundaries are evaluated from the color excess $E(B-V)$ and astrometric data in the Hipparcos catalog (Perrvman (1997)), after first ignoring stars with variability as shown by the Hipparcos index I $>0$. The ISM close to Sco-Cen stars, and associated with the $18.5^{\circ}$ tilt of Gould's Belt, are within the range of $|\mathrm{Z}|<50 \mathrm{pc}$.
} 
of local interstellar clouds is flowing past the Sun. Clouds with densities of $\approx 0.3 \mathrm{~cm}^{-3}$ and $\approx 10^{3} \mathrm{~cm}^{-3}$ have been identified in this flow. Despite the large difference in scale sizes between the heliosphere, with a distance to the upwind heliopause of $\sim 150 \mathrm{AU}$ $(0.0007 \mathrm{pc})$, and the Local Bubble, the heliosphere traces the solar environment that is set by the Local Bubble interior, in particular the interstellar radiation field and magnetic field. The Local Bubble environment of the Sun affects the past, present, and future heliosphere boundary conditions.

The heliosphere varies over geologically short timescales due to the velocities of the Sun and wispy local interstellar clouds through space. Fig. 1 shows the vector motion of the Sun through the interior of the Local Bubble, based on the solar apex motion through the local quasi-inertial frame known as the local standard of rest (LSR, we use a solar velocity of $18.0 \pm 0.9 \mathrm{~km} \mathrm{~s}^{-1}$, towards $\ell, b=47.9^{\circ} \pm 3.0^{\circ}, 23.8^{\circ} \pm 2.0^{\circ}$, based on results in Schönrich et al. 2010).

The heliospheric boundary conditions are set by the CISM. Interstellar neutral gas and dust are replenished in the heliosphere over timescales of $\sim 30$ years due to the relative $26 \mathrm{~km} \mathrm{~s}^{-1}$ motion of the Sun and CISM. Self-consistent photoionization models of the surrounding ISM provide a good match to data on the ISM inside and adjacent to the heliosphere (Slavin and Frisch 2008, S08). 2 These models predict densities and temperatures for the CISM of $n\left(\mathrm{H}^{\circ}\right)=0.19 \mathrm{~cm}^{-3}, n_{\mathrm{e}}=0.065 \mathrm{~cm}^{-3}, n_{\mathrm{p}}=0.055 \mathrm{~cm}^{-3}$, and $\mathrm{T}=6,300 \mathrm{~K}$ (from Model 26 in SF08). The fractional ionizations of $\mathrm{H}$ and He are $22 \%$ and $39 \%$, respectively. Constraints on the models include the density and temperature of interstellar He inside of the heliosphere, the local ISM towards $\epsilon$ CMa (and Sirius), pickup ion data giving cloud neutrality, and an interstellar radiation field that includes ultraviolet, soft X-ray, and extreme ultraviolet emissions, including contributions from a conductive boundary on the local interstellar cloud. Regardless of whether the Local Bubble plasma contains hot plasma, the models and data indicate that the CISM is warm, partially ionized and low density (see Model 42 in SF08).

The ISM within $\sim 30 p c$ has been shocked, since the gas-phase abundances are characteristic of the pattern expected from the destruction of refractory grains in 50$100 \mathrm{~km} \mathrm{~s}^{-1}$ shock fronts (Frisch et al. 1999). An association of the local ISM with an evolved superbubble shell is thus suggested. The abundances for the refractory elements $\mathrm{Mg}, \mathrm{Si}$, and $\mathrm{Fe}$ are larger by factors of $4-8$ than abundances of a cold cloud, and $\mathrm{C}$ is overabundant. Comparisons of interstellar gas-phase $\mathrm{Mg}, \mathrm{Fe}$, and $\mathrm{Si}$ abundances with solar abundances show that the local grains are olivine silicates, that can be efficiently aligned by magnetic fields (Frisch et al. 2011).

The properties of the circumheliosphere ISM have varied rapidly over time. LSR velocities of the Sun and most nearby interstellar clouds are near $18 \mathrm{~km} \mathrm{~s}^{-1}$ (or $\approx 18$ pc Myrs $^{-1}$ ); however the cluster of local interstellar clouds (CLIC, within $30 \mathrm{pc}$ ) is part of a general flow of ISM past the Sun so that many relative Sun-cloud velocities are larger than $14 \mathrm{~km} \mathrm{~s}^{-1}$ (Table 1). The LSR vector motion of the CLIC is -16.8 $\mathrm{km} \mathrm{s}^{-1}$ arriving from the upwind direction $\ell \sim 335^{\circ}, b \sim-7^{\circ}$ (Figure 1). This direction is towards the center of the Loop I (North Polar Spur) supernova remnant (at $\ell, b=320^{\circ}, 5^{\circ}$, Heiles 1998), and makes an angle of $70^{\circ} \pm 35^{\circ}$ with the direction of the local interstellar magnetic field (Frisch et al. 2010). These relative Sun-CLIC veloci-

2 Note there are different velcity models for the Local Interstellar Cloud (LIC, Lallement et al. 1995; (Frisch et al. 2002; Redfield and Linskv 2008); we assume that the ISM inside of the heliosphere is part of the LIC seen towards Sirius, $2.7 \mathrm{pc}$, and $\epsilon$ CMa. Recent IBEX observations are consistent with the conclusion that the Sun is in the LIC cloud seen towards Sirius (see Frisch and McComas, this volume). 

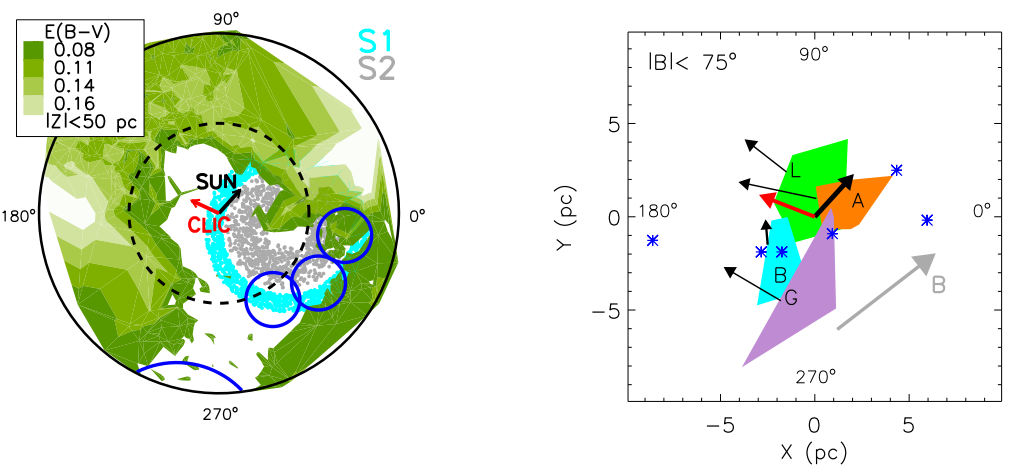

Fig. 1 Left: The distribution of interstellar dust within $200 \mathrm{pc}$ of the Sun and $50 \mathrm{pc}$ of the galactic plane, according to the cumulative amount of ISM traced by color excess E(B-V). The solar and CLIC motions through the LSR are given, respectively, by the thick black and red arrows (in both figures). The intersection of the S1 (cyan) and S2 (gray) magnetic shells of Loop I with the galactic plane are shown based on spherical shells from Wolleben (2007). The $E(B-V)$ contour levels of $0.08,0.11,0.14,0.16 \mathrm{mag}$ correspond to $N\left(\mathrm{H}^{\circ}+\mathrm{H}_{2}\right)$ column densities of $4.46 \mathrm{e} 20,6.56 \mathrm{e} 20,7.87 \mathrm{e} 20$, and $9.18 \mathrm{e} 20 \mathrm{~cm}^{-2}$, for $N\left(\mathrm{H}^{\circ}+H_{2}\right) / E(B-V)=5.8 \mathrm{e} 21$ atoms $\mathrm{cm}^{-2} \mathrm{mag}$ (Bohlin et al. $(1978)$ ). The large blue circles show the three subgroups of the Sco-Cen Association; the arc centered near $\ell \sim 260^{\circ}$ shows the approximate nearside of the Gum Nebula. Right: The locations of the nearest stars in Table 1 are plotted in the galactic plane, together with the interstellar magnetic field direction (gray arrow, from Frisch et al. 2010), and interstellar clouds within 5 pc of the Sun and $75^{\circ}$ of the galactic plane. The clouds that are plotted include the LIC and the Blue clouds (green, blue, from RL08), and the G-cloud and Apex clouds (lavender and orange, from Frisch 2003). The clouds are labeled, and LSR velocities are shown.

ties and the configuration of local ISM (Figure 1) indicate that the Sun has recently emerged from the hot Local Bubble plasma and entered the CLIC.

Cloud velocity is a key interstellar variable affecting the heliosphere boundary conditions, because the interstellar ram pressure varies as the square of the Sun-cloud velocity. The CLIC is a decelerating flow. From upwind to downwind, cloud velocities relative to the Sun $\left(V_{\mathrm{HC}}\right)$ are $-28.4 \mathrm{~km} \mathrm{~s}^{-1}$ towards $36 \mathrm{Oph}, 26.3 \mathrm{~km} \mathrm{~s}^{-1}$ in the inner heliosphere (according to Möbius et al. 2004), and $23.4 \mathrm{~km} \mathrm{~s}^{-1}$ towards $\chi^{1}$ Ori (Table 1). For otherwise similar clouds, these velocity differences lead to a $50 \%$ difference in the ram pressures of the ISM on the heliosphere over timescales of less than $\approx 4000$ years. Over longer timescales of $10^{5}$ years, the heliocentric cloud velocities and cloud lengths in Table 1 suggest variations of a factor of $\sim 4$ in interstellar ram pressures.

The distribution of the low column density local clouds, $N\left(\mathrm{H}^{\circ}\right)<10^{18.5} \mathrm{~cm}^{-2}$, is patchy. The volume density of neutral gas in nearby clouds is known only for the CISM. If all local clouds have this same density, $n\left(\mathrm{H}^{\circ}\right)=0.2 \mathrm{~cm}^{-3}$, then $\sim 35 \%$ of the sightlines to stars within $10 \mathrm{pc}$ are filled with warm low density ISM. The clouds have a mean thickness $0.9 \pm 0.3 \mathrm{pc}$, and the Sun would cross these clouds with a mean crossing time of $\sim 47,000$ years.

Magnetic fields permeate the region of the CLIC, and create asymmetries in the heliosphere configuration. The Sun appears to be located in or near the rim of the "S1" magnetic shell associated with Loop I (e.g. Wolleben 2007; Frisch 2010). Magnet- 
ically aligned interstellar dust grains near the Sun create a birefringent medium with lower optical opacities for directions parallel to the interstellar magnetic field (ISMF), and therefore polarize starlight. The direction of ISMF over the nearest $40 \mathrm{pc}$ has been found from fits to the position angles of polarized starlight, giving a direction towards $\ell, b=38^{\circ}, 23^{\circ}$, with uncertainties of $\sim \pm 35^{\circ}$ (Frisch et al. 2010). The $\sim 70^{\circ}$ angle between the magnetic field and upwind LSR CLIC direction is consistent with an ISMF compressed in an expanding superbubble shell. The ISMF direction indicated by the Interstellar Boundary Explorer (IBEX) Ribbon arc center, $\ell, b=33^{\circ}, 55^{\circ}$ (Frisch and McComas 2010, this volume) is consistent with the polarization direction to within uncertainties. A more distant measure of the ISMF is provided by fits to the Faraday rotation and dispersion measures for four pulsars, 150-300 pc away in the third galactic quadrant, which give a ISMF direction similar to the polarization value, and indicate a field strength of $\sim 3.3 \mu \mathrm{G}$ (Salvati 2010).

Not all nearby clouds are warm and diffuse. Towards the constellation of Leo, and within $12 \mathrm{pc}$, a tiny dense cold filamentary interstellar cloud with thickness $<0.4 \mathrm{pc}$ has been identified in $\mathrm{Na}^{\circ}$ absorption (Meyer et al. 2006). This cloud is similar to the tiny scale atomic structures (TSAS) that are observed throughout the ISM, with typical sizes $30 \mathrm{AU}$, volume densities $\sim 10^{3} \mathrm{~cm}^{-3}$, column densities $10^{18}-10^{19} \mathrm{~cm}^{-2}$, and thermal pressures $P / k=n T=10^{4}-10^{6} \mathrm{~cm}^{-3} \mathrm{~K}$ (Stanimirovic 2009). Once the solar motion is removed from cloud velocities (Figure 1, right), the small Blue cloud is also seen to form in a region where the LIC and G-cloud are colliding. Both the Leo cloud and the Blue cloud (e.g. HD 80007) coincide with the ring of tiny dense cold clouds identified in $\mathrm{H}^{\circ} 21-\mathrm{cm}$ by (Haud 2010). The CLIC is a decelerating flow of ISM, so that a scenario where the upwind G-cloud and downwind LIC are converging is consistent with the velocity data. Evidently tiny cold clouds can and have formed very close to the Sun through cloud collisions, so that the recent paleoheliosphere may well have included intervals where galactic cosmic ray modulation was minimal (see next section).

Other possible types of nearby ISM include conductive, evaporative, boundaries between warm diffuse gas and hot plasma between the clouds. Unsaturated outflows of ISM from the clouds lead to sharp gradients in the ISM density, velocity, and temperature over small spatial scales. The outflow velocity can be up to $20 \mathrm{~km} \mathrm{~s}^{-1}$, with temperature variations of an order of magnitude, over distances $<0.5 \mathrm{pc}$ (SF08).

\subsection{Configuration of Local ISM}

Our goal is to explore the connection between the paleoCISM and the paleomagnetic record on Earth. This connection is possible because of the heliosphere response to variations in the circumheliospheric ISM, and the cosmic ray flux at Earth is connected both to the heliosphere and neutrality of surrounding ISM. Optimally we need a model of the properties of nearby clouds, including cloud shapes, density, ionization, velocity, and homogeneity. Most of these data are unavailable, so we rely on simple assumptions for the cloud configuration and density.

Discussions of the effect of the ISM on the heliosphere will be restricted to clouds that extend to within $5 \mathrm{pc}$ of the Sun. For a typical Sun-cloud velocity of $17 \mathrm{~km} \mathrm{~s}^{-1}$, this would correspond to a look-back time of $\sim 300,000$ years. The most complete data set available for determining cloud configurations are the $\mathrm{H}^{\circ}$ and $\mathrm{D}^{\circ}$ data, which are free of uncertainties regarding elemental abundances (Redfield and Linsky 2004a; 
Wood et al. 2005). Column densities for $\mathrm{H}^{\circ}$ or $\mathrm{D}^{\circ}$ can be transformed to distance scales characteristic of the neutral gas for some assumed volume density for $\mathrm{H}^{\circ}$. We will assume that nearby clouds have the same neutral density as the LIC, $n\left(\mathrm{H}^{\circ}\right)=0.2$ $\mathrm{cm}^{-3}$.

Clouds near the Sun are typically identified through parsing absorption line velocity data into different clouds by assuming rigid-body motion through space for each cloud. A number of studies provide data on cloud velocities, but the most complete study is that of Redfield and Linskv (2008, RL08). However, one of the conclusions of this study, that the LIC does not surround the heliosphere, may change with better measurements of the velocity of interstellar $\mathrm{He}^{\circ}$ inside of the heliosphere (Frisch and McComas, this volume). Distinct interstellar "clouds" have been found within 5 pc in a number of different studies (e.g. see review Frisch et al. 2011). We adopt the LIC and Blue cloud (B) properties from RL08, and the G cloud (G) and Apex cloud (A) from (Frisch 2003); additional column density data is provided by Wood et al. (2005) and Redfield and Linsky (2004a). The configuration of these clouds, within $60^{\circ}$ of the galactic plane, is shown in Figure 1, right. The LSR velocity vectors for the clouds are shown, together with those of the CLIC and Sun. For the purposes of estimating cloud thicknesses from $\mathrm{Ca}^{+}$, the conversion factor of $N\left(\mathrm{Ca}^{+}\right) / N(\mathrm{HI})=1.5 \times 10^{-8}$ is adopted from $\alpha$ Aql and $\eta$ UMa data.

Estimates of the times it took (or will take) for the Sun to cross interstellar clouds observed towards several nearby stars are listed in Table 1, for the simplest assumption that the time is given by $T=L / V_{\mathrm{HC}}$, for $L=N\left(\mathrm{H}^{\circ}\right) / n\left(\mathrm{H}^{\circ}\right)$, heliocentric velocity $V_{\mathrm{HC}}$, and $n\left(\mathrm{H}^{\circ}\right)=0.2 \mathrm{~cm}^{-3}$. For stars near the upwind or downwind directions, this assumption should give realistic estimates as long as the cloud is homogeneous and neutrals and ions are well mixed. Obviously irregular cloud shapes, or cloud motions from unmeasured non-radial velocities, particularly for sightlines parallel to cloud surfaces or for inhomogenous clouds, can affect these basic estimates. Also listed in Table 1 are the angles between each star and the upwind directions of the vector CLIC, and vector LIC (from $\mathrm{He}^{\circ}$ ), velocities through the LSR. Whether clouds in the local ISM are filamentary or blobs affects the time inferred for the Sun to cross the cloud, particularly for stars making a large angle with the upwind direction where radial velocities are small.

The times in Table 1 are estimates only, since accurate dating of solar transitions of cloud boundaries requires data on the volume densities that are not generally available. If the clouds are in thermal pressure equilibrium, the cloud temperature variations of a factor of two or more (Redfield and Linskv 2004b) suggest there are also variations in cloud densities, which can not be evaluated without information on cloud ionization. In addition, the structure of local ISM is resolved at very low spatial resolution, since current data only sample the sky at a spacing of $\sim 1$ star per 260 square-degrees (e.g. RL08).

\subsection{Possible Historical Changes in the Circumheliospheric ISM}

Stars located close to the downwind directions of cloud velocities will give the best estimates for entry times into the cloud, for simple geometries. For the LIC, downwind corresponds to small values for $\phi_{\mathrm{HeI}}$. Estimates of the time when the Sun entered in the LIC will be based on interstellar data towards four stars, two near the downwind direction of the LIC ( $\chi^{1}$ Ori and $\alpha$ Aur), and two at more oblique angles to the LIC 
Table 1 Crossing times for Clouds Close to Sun ${ }^{(1)}$

\begin{tabular}{lcccccc}
\hline Star & $\ell, b$, Dist, Cld & $V_{\mathrm{HC}}, V_{\mathrm{LSR}}$ & $N\left(\mathrm{H}^{\circ}\right)$ & $\begin{array}{c}L, \text { Cloud } \\
\text { Thick. } \\
(\mathrm{pc})\end{array}$ & $\begin{array}{c}\theta_{\mathrm{CLIC}}, \phi_{\mathrm{HeI}} \\
\text { LSR, HC } \\
(\mathrm{deg})\end{array}$ & $\begin{array}{c}T, \text { Crossing } \\
\text { Time } \\
(\text { years })\end{array}$ \\
\hline$\alpha \mathrm{Cen}$ & $316,-1,1.3, \mathrm{G}$ & $-18.0,-18.6$ & 17.61 & 0.66 & $20^{\circ}, 130^{\circ}$ & $(35,900)$ \\
$\alpha \mathrm{CMa}$ & $227,-9,2.7, \mathrm{~L}$ & $19.6,2.2$ & 17.60 & 0.65 & $106^{\circ}, 42^{\circ}$ & 32,200 \\
$\alpha \mathrm{CMa}$ & $227,-9,2.7, \mathrm{~B}$ & $13.7,-3.7$ & 17.40 & 0.41 & $106^{\circ}, 42^{\circ}$ & 29,000 \\
$\alpha \mathrm{CMi}$ & $214,13,3.5$ & $24.0,10.4$ & 17.81 & 1.86 & $122^{\circ}, 41^{\circ}$ & 42,600 \\
$\alpha \mathrm{CMi}$ & $214,13,3.5, \mathrm{~L}$ & $20.5,6.5$ & 18.08 & 1.38 & $122^{\circ}, 41^{\circ}$ & 92,900 \\
$70 \mathrm{Oph}$ & $30,12,5.1, \mathrm{G}$ & $-26.2,-9.3$ & 17.77 & 0.95 & $58^{\circ}, 26^{\circ}$ & $(670,000)$ \\
$70 \mathrm{Oph}$ & $30,12,5.1, \mathrm{~A}$ & $-32.4,-15.5$ & 17.46 & 0.47 & $58^{\circ}, 26^{\circ}$ & $(530,000)$ \\
$\alpha \mathrm{Aql}$ & $48,-9,5.1, \mathrm{G}$ & $-18.1,-0.3$ & 17.91 & 1.3 & $72^{\circ}, 50^{\circ}$ & $(1.1 \mathrm{e} 6)$ \\
$\alpha \mathrm{Aql}$ & $48,-9,5.1, \mathrm{~A}$ & $-26.9,-10.7$ & 17.47 & 0.48 & $72^{\circ}, 50^{\circ}$ & $(670,000)$ \\
$\chi^{1} \mathrm{Ori}$ & $188,-3,9, \mathrm{~L}$ & $22.3,27.4$ & 17.93 & 1.38 & $146^{\circ}, 13^{\circ}$ & 60,500 \\
$\alpha \mathrm{Aur}$ & $163,4,13, \mathrm{~L}$ & $21.8,15.3$ & 18.24 & 2.82 & $171^{\circ}, 28^{\circ}$ & 126,000 \\
$36 \mathrm{Oph}$ & $358,7,6, \mathrm{G}$ & $-28.2,-16.7$ & 17.85 & 1.15 & $26^{\circ}, 170^{\circ}$ & $(39,800)$ \\
\hline
\end{tabular}

(1) Column 1 gives the star number in Figure 1, and star name. The galactic coordinates, distance, and cloud to which the component is attributed, are listed in Column 2. G and A refer to the G-cloud and Apex cloud (from Frisch 2003), and L and B refer to the LIC and Blue clouds (from Redfield and Linskv 2008). Column 3 gives the observed velocities of the interstellar absorption components in the solar (e.g., Redfield and Linsky (2008)) and LSR inertial systems Column 4 is the log of the cloud column density, from Wood et al. (2005) or Hebrard et al. (1999). Column 5 is the cloud thickness, $N\left(\mathrm{H}^{\circ}\right) / n\left(\mathrm{H}^{\circ}\right)$ calculated for volume density $n\left(\mathrm{H}^{\circ}\right)=0.2 \mathrm{~cm}^{-3}$. Column 6 gives the angles $\theta_{\mathrm{CLIC}}$ and $\phi_{\mathrm{HeI}}$, which are the angles between the star and the LSR upwind CLIC direction $\left(\ell, b=335^{\circ},-7^{\circ}\right)$, and the heliocentric (HC) downwind $\mathrm{He}^{\circ}$ vector $\left(\ell, b=184^{\circ},-15^{\circ}\right)$, respectively. Column 7 gives a nominal crossing time for the cloud, calculated from the cloud thickness and $V_{\mathrm{HC}}$. The times inside of parentheses are in the future.

vector ( $\alpha \mathrm{CMi}$, and $\alpha \mathrm{CMa}$ ). The Sun is assumed to be in the LIC. Because of the uncertainties in the ionization, volume density, magnetic pressure, an homogeneity of these clouds, the timescales below are quite uncertain. However they do show the plausibility of an interstellar effect on the cosmogenic isotope record.

The star $\chi^{1}$ Ori, at $9 \mathrm{pc}$, is viewed through the heliosphere tail $\left(\phi_{\mathrm{HeI}}=13^{\circ}\right.$, Table 1). The observed $V_{\mathrm{HC}}=22 \mathrm{~km} \mathrm{~s}^{-1}$ component corresponds to the LIC velocity. For the above premise that $L=N\left(\mathrm{H}^{\circ}\right) / n\left(\mathrm{H}^{\circ}\right)$, the Sun would have entered the LIC 60,500 years ago. The relatively high turbulence of the LIC absorption lines towards $\chi^{1}$ Ori (e.g. Redfield and Linsky 2008), however, suggests that two clouds with similar velocities could blend in velocity to form this component. If so, the Sun would have entered the LIC more recently. A more distant star in the downwind direction is $\alpha$ Aur $\left(\phi_{\mathrm{HeI}}=28^{\circ}\right)$. The higher LIC $\mathrm{H}^{\circ}$ column densities suggest, instead, that the the Sun entered the ISM 126,000 years ago. The star $\alpha \mathrm{CMi}$ is much closer to the Sun, but it is offset $41^{\circ}$ from the downwind $\mathrm{LIC}$ direction $\left(\phi_{\mathrm{HeI}}=41^{\circ}\right)$. The LIC component gives an entry time into the LIC of 92,900 years ago.

The closest downwind star is Sirius ( $\alpha \mathrm{CMa}, 2.7 \mathrm{pc}$ ), with two clouds in front of it. Sirius is $106^{\circ}$ from the downwind direction. The Sun would have entered the LIC component 32,200 years ago, and the Blue cloud $\sim 29,000$ years before that.

An alternate LIC encounter time follows from the assumption that the LIC is filamentary, with the filament oriented perpendicular to the LSR velocity and roughly parallel to the magnetic field direction. When distant diffuse ISM is spatially resolved, 
filamentary structures are generally observed for neutral and ionized gas. Magnetic pressure creates filamentary ISM towards Loop I at distances of $\sim 90 \mathrm{pc}$, so the evident relation between Loop I and nearby ISM suggest the LIC could also be filamentary. Frisch (1994) assumed a filamentary structure for the LIC, with the direction of the LSR cloud velocity perpendicular to the filament, and found the Sun may have entered the CISM gas anytime within the past 2,000-8,000 years, or even more recently.

For the comparisons with the geologic radio isotope record in Section 4, and since the detailed structure of the downwind gas is not known, we adopt several time intervals as candidates for a solar transition between interstellar clouds. The primary downwind clouds of interest are the LIC, which is most likely presently surrounding the Sun, and the Blue cloud (Figure 1, right). These transitions are: (1) Sometime within the past 8,000 years, as indicated by the $\alpha$ CMa sightline together with an assumed filamentary cloud structure. (2) Sometime within 10,000 - 32,000 years ago. (3) Before that the Sun was in the Blue cloud, with an entry time of $\sim 61,000$ years ago from the $\alpha$ CMa timescales. The Blue cloud should be more ionized than the LIC because it is less shielded from the primary source of local H-ionization, $\epsilon$ CMa. This suggests that the Sun traveled through a cloud with higher ionization levels than the LIC, before it entered the LIC. This interpretation is also consistent with the local ionization gradient found by Wolff et al. (1999). (4) Any of the surfaces of these clouds may have a conductive interface. However the clumpiness of the local ISM, and the apparent deceleration evident in the CLIC velocities, suggest that the clumps of gas that form the CLIC have a cohesive origin and are close to each other. Transition times across $0.5 \mathrm{pc}$ cloud interfaces, where the relative Sun-cloud velocities may be larger by $20 \mathrm{~km} \mathrm{~s}^{-1}$, may be 10,000 years, or less, depending on the angle of the ISMF that inhibits conduction. (5) No tiny dense clouds have been directly detected nearby in the downwind direction, however the Blue cloud coincides with a ring of tiny cold dense clouds (Section 2.1). Typical sizes for these clouds, as small as 30 AU, indicate that such clouds would sweep past the heliosphere over timescales of a decade.

\section{The Heliosphere for Different Interstellar Environments}

The heliosphere results from a balance between solar wind and interstellar pressure. This balance sets the size of the heliosphere and determines the particle distributions and magnetic field configurations throughout, which in turn determine how galactic cosmic rays are being modulated during their passage through the heliosphere on their way to Earth.

The inner boundary conditions of the heliosphere consist of the solar wind, imprinted with the 22 year magnetic activity cycle of the Sun, as a "point source" inflow. The time variability of the solar wind affects the large-scale heliosphere, including the heliosheath regions immediately inside and outside of the heliopause. The distance of the termination shock in the nose direction varies by about $10 \mathrm{AU}$ with the solar cycle phase, and is closer during solar wind minima when ram pressures are lower (Zank and Müller 2003). The reversals of the solar magnetic polarity every $\sim 11$ years between successive solar minima propagate to the inner heliosheath regions, and create bands of magnetic polarity that are swept around the flanks of the heliosphere with the subsonic solar wind (e.g., Pogorelov et al. 2009). The outer boundary conditions of the heliosphere are dominated by the total pressure of the surrounding cloud, including magnetic pressure, the ram pressure of excluded ions, and a large fraction of the ram 

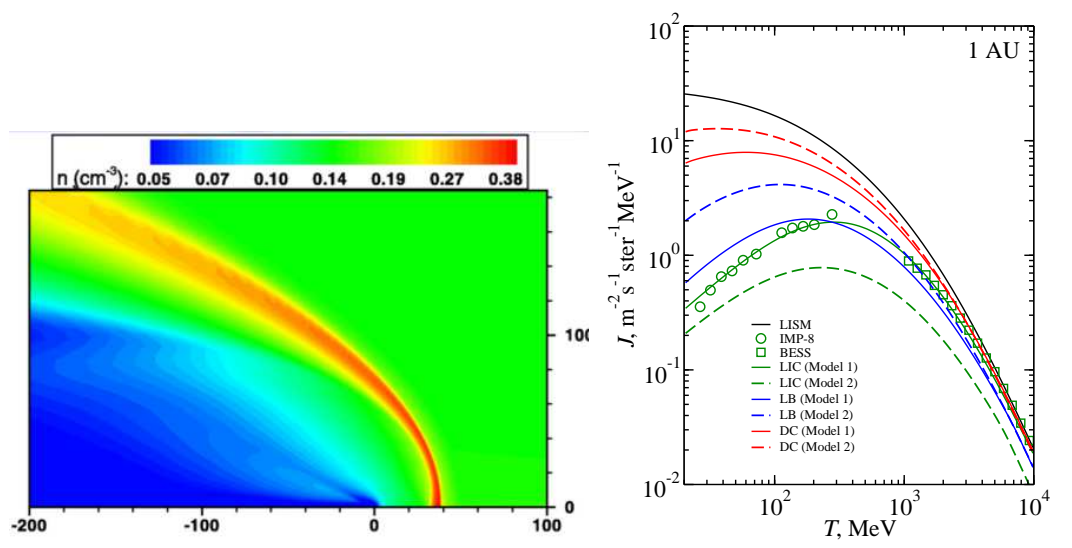

Fig. 2 Left. The density distribution for the heliosphere when immersed in a moderately dense cloud, $n\left(\mathrm{H}^{\circ}\right) \sim 15 \mathrm{~cm}^{-3}$, with a temperature $\mathrm{T}=3,000 \mathrm{~K}$, ion density of $0.2 \mathrm{~cm}^{-3}$, and moving at the LIC velocity (Model 17 in Müller et al. 2006). The heliopause is 34 AU from the Sun. Right. Spectra of galactic cosmic rays at $1 \mathrm{AU}$ for three different interstellar clouds surrounding the heliosphere (ACRs are not included). LIC stands for the contemporary CISM; LB is the Local Bubble interior modeled as a $1.2 \times 10^{6} \mathrm{~K}$ fully ionized plasma with density $0.005 \mathrm{~cm}^{-3}$; and DC is a dense cloud with density $10 \mathrm{~cm}^{-3}, \mathrm{~T}=200 \mathrm{~K}$, and relative velocity of $25 \mathrm{~km} \mathrm{~s}^{-1}$. Models 1 and 2 utilize different cosmic ray modulation models (figure from Florinski and Zank 2006).

pressure of the neutrals which participate through charge-exchange with ions outside the heliopause; the pressure interior of the heliopause is modified by charge exchange as well.

Configurations of the global heliosphere have been modeled for a range of surrounding interstellar cloud properties. In one study (Müller et al. 2008), the parameter space around the current interstellar cloud is sampled to establish empirical relations for locations of heliopause, termination shock, and bow shock as a function of interstellar boundary values. This study confirms the overall connection of the size of the heliosphere with the pressure balance argument. The interstellar ionization ratio weakly influences the downstream termination shock distance as well as the typical termination shock strength. In relative terms, the termination shock shape does not change in this particular region of parameter space. Müller et al. (2006) explore a wide range of possible interstellar environments, including cold dense clouds, hot tenuous, completely ionized clouds like that assumed for the Local Bubble, and systems on galactic paths that result in fast relative Sun-ISM velocities. In particular the relative velocity has a decisive influence on how much interstellar neutral material reaches the inner heliosphere (filtration); the larger the relative velocity, the less filtration is occurring. If the Sun encounters a completely ionized ISM, such as the Local Bubble, GCR particle fluxes at Earth are lower than currently. With neutrals absent, none of the pressure balance modifications take place, and secondary particles like anomalous cosmic rays (ACR) do not exist. Lastly, if the heliosphere is embedded in a dense cloud, the heliosphere is small in size, and particle fluxes at Earth rise substantially. Depending on the interstellar ram pressure, the heliosphere can easily get small enough for the Earth orbit to be partly in the inner heliosheath region. 
Yeghikvan and Fahr (2006) consider the passage of very dense molecular clouds over the heliosphere. The resulting heliospheres are very small, so that the orbit of Earth takes it through regions of interstellar gas outside the heliopause. The expected neutral fluxes at Earth are so high that changes for the ozone layer and other climaterelated effects are to be expected. Similarly, when a supernova shock front washes over the heliosphere (Müller et al. 2009), the heliosphere can be equally small (Figure 2a), with direct access of supernova material to the Earth atmosphere. In both cases of extremely small heliospheres, the access of GCR to Earth is greatly enhanced. In a calculation by Florinski et al. (2003), where a cold dense cloud results in a moderate size heliosphere of $23 \mathrm{AU}$, a two-fold flux of GCR, and a ten-fold flux of ACR is reported. Figure $2 \mathrm{~b}$ illustrates this with calculated spectra for the Local Bubble case and for a dense cloud case, comparing them to the GCR fluxes of the contemporary heliosphere.

\section{Comparing past cloud transitions with the terrestrial radioisotope record}

Spallation of cosmic-rays in the terrestrial atmosphere creates the radioisotopes ${ }^{10} \mathrm{Be}$, ${ }^{36} \mathrm{Cl}$, and ${ }^{14} \mathrm{C}$ that are used to date the repository geological archive. Both the geomagnetic and heliosphere magnetic fields modulate the incident cosmic-ray flux (e.g. McCracken 2004, and the Beer and McCracken articles in this volume), including the ACR component. The well known anticorrelation between cosmic ray fluxes at $1 \mathrm{AU}$ and the solar magnetic activity cycle (see articles by Leske and Mewaldt in this volume) suggests that ISM-driven variations in the heliosphere may also be significant. Earlier interstellar explanations for the peaks in the concentration of ${ }^{10} \mathrm{Be}$ in the ice core record include the reduced modulation of cosmic rays by a heliosphere that has been compressed by a passing supernova shock (Sonett et al. 1987, and see Fig. 2), and higher GCR fluxes incident on the heliosphere because the Sun traveled through a magnetic flux tube in the ISM (Frisch 1997). High GCR fluxes have also been linked to climate cooling (e.g., Kirkby and Carslaw 2006).

The paleomagnetic field that is derived from geological data such as magnetic remenance should, in principle, agree with the paleomagnetic field that has been derived from the radioisotope ${ }^{10} \mathrm{Be}$ and ${ }^{36} \mathrm{Cl}$ ice core data, and the ${ }^{14} \mathrm{C}$ tree-ring data. Reconstructing the geomagnetic field from cosmogenic isotope data requires an assumption about the flux and spectra of cosmic rays, and the production and dispersal of radioisotopes. Muscheler et al. (2005, M05) compared the paleomagnetic field determined by these different techniques, and found several discrepancies that are not explained. Given the mystery of these anomalies, we postulate that temporal variations in the properties of the ISM shaping the heliosphere led to variations in the total cosmic-ray spectrum at the Earth. These variations have been overlooked as a factor in the geomagnetic time-line developed from cosmogenic isotopes, but need to taken into consideration.

The anomalies in the reconstructed paleomagnetic record mentioned in the Muscheler et al. (2005) paper, and that are discussed here are: 1. The discrepancy in the timing of the maximum geomagnetic field dipole strengths (the "virtual axis dipole moment", VADM) during the late Holocene traced by the ${ }^{10}$ Be versus ${ }^{14} \mathrm{C}$ records (see Fig. 6 in M05). 2. The interval of $18,000-34,000$ years ago, where both ${ }^{10} \mathrm{Be}$ and ${ }^{36} \mathrm{Cl}$ VADM reconstructions show increased modulation compared to the remanence records, and the ${ }^{14} \mathrm{C}$ data are poorly understood. 3 . An anomaly 48,000 years ago where both ${ }^{10} \mathrm{Be}$ and 
${ }^{36} \mathrm{Cl}$ showed increased modulation compared to the VADM record, and an anomaly 58,000 years ago where only ${ }^{36} \mathrm{Cl}$ shows increased modulation.

Cosmic ray fluxes at Earth vary with the size and properties of the heliospheric modulation region. A second simple change in the CISM properties, that would necessarily affect cosmic ray fluxes at the Earth, would be spatial variations in the ionization levels of the interstellar gas. At $1 \mathrm{AU}$ the cosmic-ray fluxes below $\sim 200 \mathrm{MeV} /$ nucleon are dominated by anomalous cosmic rays (ACRs). For instance, the ACR oxygen intensity of $10 \mathrm{MeV} /$ nucleon is an order of magnitude larger than for GCR oxygen (Leske et al. 2008). ACRs form from neutral interstellar atoms that charge-exchange with the solar wind, creating pickup ions that are accelerated to cosmic ray energies. The ACRs will vanish if the surrounding ISM is ionized (with the exception of a very minor contribution arising from dust-generated pickup ions). These spatial variations translate to temporal variations in the $1 \mathrm{AU}$ cosmic ray fluxes because of the relative Sun-cloud motion.

Models of radioisotope production include the GCR sources, and cosmic rays from energetic solar flares. However, they do not distinguish ACRs as a source with a distinct origin and spectrum, and one that is likely to vary as the Sun encounters different clouds. Radioisotope production has been calculated for solar cosmic rays at similar energies as ACRs (Webber et al. 2007), but not for the ACR spectrum itself. ACRs are trapped in the radiation belts of Earth (Mewaldt et al. 1998).

The primary question then becomes: How would the ${ }^{14} \mathrm{C},{ }^{10} \mathrm{Be}$, and ${ }^{36} \mathrm{Cl}$ production change as the ACR component vanishes? Three possible effects may contribute. The first is that ${ }^{14} \mathrm{C}$ is formed by thermalized neutrons near the top of the atmosphere, and the long storage time ( $>0.1$ year) of ACRs trapped in the radiation belts of Earth (Mewaldt et al. 1998) may multiply the production of ${ }^{14} \mathrm{C}$ because of the long exposure times of nitrogen and carbon compounds in the atmosphere compared to the direct production of cosmogenic isotopes formed by spallation. An opposite effect of ACRs on cosmogenic isotope production is suggested by the yield functions of ${ }^{10} \mathrm{Be}$ and ${ }^{14} \mathrm{C}$ as a function of the energy of the incident cosmic ray proton, which are shown in Figure 6 in Usoskin (2008). The production of ${ }^{10} \mathrm{Be}$ is relatively more efficient at ACR energies then the production of ${ }^{14} \mathrm{C}$, based on comparisons of the ratios of production at $\sim 200 \mathrm{MeV}$ (ACR energies) and $\sim 20 \mathrm{GeV}$ (GCR energies). These ratios for ${ }^{10} \mathrm{Be}$ and ${ }^{14} \mathrm{C}$ are $\sim 0.03$ and $\sim 0.001$, respectively. These yield functions would then suggest that the production of ${ }^{14} \mathrm{C}$ is less sensitive to the ACR component of cosmic-ray fluxes than is the production of ${ }^{10} \mathrm{Be}$. A third wild-card possibility is that rapid variations of the geomagnetic field may reduce coupling between the ACRs and the radiation belts, so that ACRs have the same access to the atmosphere as GCRs, reducing any effect of storage of ACRs in the radiation belts. In the absence of a detailed understanding of the effect of ACRs on cosmogenic isotope production, the discussions below linking cloud transitions to the geological radioisotope data are highly speculative.

\subsection{Late Holocene Discrepancies and Anomalous Cosmic Rays}

The VADM paleomagnetic field reconstructed from ${ }^{10}$ Be records peaks at 2,000 years $\mathrm{BP}$, which is one millennium after the peak determined from ${ }^{14} \mathrm{C}$ at 3,000 years BP (Fig. 6 in M05). At the CHISM velocity, 3,000 years corresponds to a distance of $0.08 \mathrm{pc}$, which is a plausible value for the distance to the cloud surface in the $\alpha \mathrm{CMa}$ direction. The 2,000-3,000 time interval is within the uncertainties of the solar entry into the LIC 
as inferred from the $\alpha$ CMa sightline by Frisch (1994), based on a filamentary structure for the LIC. A LIC filamentary structure would be consistent with Figure 1, right, only if the density structure of the ISM moving at the LIC velocity is inhomogeneous.

Since VADM variations anticorrelate with radioisotope fluxes, the ${ }^{14} \mathrm{C}$ fluxes decreased a millennium before the ${ }^{10}$ Be fluxes decreased in the Holocene. Muscheler et al. (2005) postulated that this discrepancy was due to the carbon cycle, because the ${ }^{10} \mathrm{Be}$ and archeomagnetic field determinations generally agree.

If the discrepancy is due, instead, to variations in the ISM, we suggest that ACRs may be the culprit. The three possible influences of ACRs on the cosmogenic isotope record leads to three different explanations for the anomaly. Prior to entering the CISM, the Sun may have traversed a fully ionized cloud interface with no neutrals, no pickup ions or ACRs, with a larger relative Sun-cloud velocity leading to higher interstellar ram pressures (see Fig. 2 in SF08), and with reduced mass-loading of the solar wind by pickup ions. (1) If the enhanced yield of ${ }^{10} \mathrm{Be}$ production at low energies is important, the absence of ACRs at $1 \mathrm{AU}$ would increase ${ }^{14} \mathrm{C}$ relative to ${ }^{10} \mathrm{Be}$, which is not seen. (2) An opposite result follows if the long exposure times for ${ }^{14} \mathrm{C}$ formation from ACRs in the radiation belts is important, in which case an ionized ambient ISM would preferentially reduce ${ }^{14} \mathrm{C}$ levels because of the concordant lack of ${ }^{14} \mathrm{C}$ enhancement from the multiplying effect of the storage of ACRs in the magnetosphere. Once the Sun enters the main part of the cloud, which is $\sim 75 \%$ neutral, the low energy ACR component is restored and the balance between the synchronization of the ${ }^{10} \mathrm{Be},{ }^{36} \mathrm{Cl}$, and ${ }^{14} \mathrm{C}$ formation was restored along with the ACRs. (3) For the third alternative, if disruption of the terrestial magnetic field affects the ${ }^{14} \mathrm{C}$ production only, ${ }^{10} \mathrm{Be}$ would stay coupled to the geomagnetic field while the timescale for ${ }^{14} \mathrm{C}$ dating would be disrupted. The tight coupling between ${ }^{10} \mathrm{Be}$ and the geomagnetic field suggests that the overall heliospheric modulation of the GCR fluxes did not change, so that the discrepancy could be due to the effect that the disrupted geomagnetic field has on the flux of ACRs into the atmosphere.

\subsection{Cloud Crossings}

During the period $18,000-34,000$ years ago, both the ${ }^{10} \mathrm{Be}$ and ${ }^{36} \mathrm{Cl}$ VADM reconstructions deviate from the archeomagnetic remanence records. The increased VADM reconstructed from ${ }^{10} \mathrm{Be}$ and ${ }^{36} \mathrm{Cl}$ suggests reduced fluxes below that explained by the archeomagnetic record, or extra heliosphere modulation of GCRs. Muscheler et al. (2005) suggested that possible changes in levels of solar magnetic activity may be responsible for this difference. We speculate that this interval instead corresponds to an encounter between the Sun and slower clumpy ISM beyond the CISM in the downwind direction that forms the second clouds observed towards $\alpha$ CMa (the Blue cloud, Figure 1 right) and towards $\alpha$ CMi (the Aur cloud in Redfield and Linsky 2008), both within 3.5 pc. Cloud velocities alone affect the GCR modulation because the heliosphere dimensions increase as the ram pressure of the ISM decreases. The decreased ${ }^{10} \mathrm{Be}$ and ${ }^{36} \mathrm{Cl}$ fluxes in this interval would then suggest a larger heliosphere modulation region, that still contains a solar wind mass-loaded with pickup ions from interstellar neutrals. Cloud-Sun relative velocities of $26 \mathrm{~km} \mathrm{~s}^{-1}$ today, versus $21-22 \mathrm{~km} \mathrm{~s}^{-1}$ for $\chi^{1}$ Ori and $\alpha$ Aur, and $14 \mathrm{~km} \mathrm{~s}^{-1}$ for the Blue cloud towards $\alpha$ CMa (Table 1), suggest that cloud ram pressure could have been a factor of $\sim 2-3$ lower in the past than today, giving 
a larger heliosphere and larger GCR modulation region that are consistent with the decreased fluxes found by M05.

Additional discrepancies, about 46,000-48,000 and 58,000 years ago, are seen between the radio isotope paleomagnetic and remanence paleomagnetic records. If the Sun entered the neutral portion of the LIC $\sim 60,500$ years ago, as indicated by ${ }^{1} \chi$ Ori located in the downwind direction (Table 1), the VADM discrepancy suggests that increased cosmic ray modulation in the LIC, or decreased ACR production, could have produced the discrepancy. A slightly higher density, $n\left(\mathrm{H}^{\circ}\right) \sim 0.25 \mathrm{~cm}^{-3}$, would match the solar entry into the LIC to 48,000 years ago discrepancy.

\section{Future: Improving Comparisons between Local ISM Structure and Paleomagnetic Records}

Comparisons between the structure and kinematics of interstellar clouds near the Sun and the paleomagnetic records deduced from radio isotopes such as ${ }^{10} \mathrm{Be},{ }^{36} \mathrm{Cl}$, and ${ }^{14} \mathrm{C}$, are based on scanty knowledge of details of the three-dimensional spatial distribution and configuration of nearby interstellar clouds. Volume densities of both neutrals and electrons in the nearest interstellar clouds are required to improve maps of the ISM distribution. Some insight could be gleaned from photoionization models, as done for the CISM; however high-sensitivity ultraviolet data are required for realistic models.

The most significant open question is whether ACR variations are sufficient to require consideration as a separate component that contributes to the production of the cosmogenic isotopes, and that varies separately from the GCR component as the heliosphere is modulated by the ISM. One aspect of answering such questions will be to expand calculations of the fluxes of galactic and anomalous cosmic rays at the Earth beyond the limited number of heliosphere configurations now available. More study of the roles of ACRs versus higher GCRs as source populations of the radio isotopes are needed, since differences between the ${ }^{14} \mathrm{C},{ }^{10} \mathrm{Be},{ }^{36} \mathrm{Cl}$ records are mandated if ACRs are a factor in ${ }^{14} \mathrm{C}$ production rates. A better understanding of the physical properties and configuration of ISM close to the Sun is also required for accurate comparisons between the timelines of the cosmogenic isotope and heliosphere boundary conditions. Nevertheless, we conclude that in principle the attenuation of GCRs by an ISM-modulated heliosphere, and perhaps the presence or absence of ACRs, are capable of accounting for differences between the paleomagnetic record determined from cosmogenic isotopes versus remnance data.

Acknowledgements PCF thanks the International Space Sciences Institute in Bern, Switzerland, for hosting a stimulating meeting on the relation between cosmic ray fluxes and the terrestrial radio isotope record. This research has been supported in part by NASA grants NNX09AH50G and NNX08AJ33G to the University of Chicago, and by the IBEX mission as a part of NASA's Explorer Program. PCF would like to thank Ken McCracken and Jurg Beer for helpful discussions.

\section{References}

Bohlin, R. C., Savage, B. D., and Drake, J. F. (1978). A survey of interstellar H I from L-alpha absorption measurements. . Astrophys. J., 224, 132-142.

Fisk, L. A., Kozlovsky, B., and Ramaty, R. (1974). An interpretation of the observed oxygen and nitrogen enhancements in low energy cosmic rays. Astrophys. J., 190, L35-L38. 
Florinski, V. and Zank, G. P. (2006). The Galactic Cosmic Ray Intensity in the Heliosphere in Response to Variable Interstellar Environments. In Solar Journey: The Significance of Our Galactic Environment for the Heliosphere and Earth, pages 281-316 . Springer.

Florinski, V., Zank, G. P., and Axford, W. I. (2003). The Solar System in a dense interstellar cloud: Implications for cosmic-ray fluxes at Earth and ${ }^{10}$ Be records. Geophys. Res. Lett., 30, 5-1.

Frisch, P. C. (1994). Morphology and ionization of the interstellar cloud surrounding the solar system. Science, 265, 1423.

Frisch, P. C. (1997). Journey of the Sun, astro-ph/9705231 arXiv.

Frisch, P. C. (2003). Local Interstellar Matter: The Apex Cloud. Astrophys. J., 593, 868-873.

Frisch, P. C. (2010). The S1 Shell and Interstellar Magnetic Field and Gas Near the Heliosphere. Astrophys. J., 714, 1679-1688.

Frisch, P. C. and McComas, D. J. (2010). The Interstellar Boundary Explorer (IBEX): Tracing the Interaction between the Heliosphere and Surrounding Interstellar Material with Energetic Neutral Atoms. Space Sci. Rev., submitted, 00, 0-0.

Frisch, P. C., Dorschner, J. M., Geiss, J., Greenberg, J. M., Grün, E., Landgraf, M., Hoppe, P., Jones, A. P., Krätschmer, W., Linde, T. J., Morfill, G. E., Reach, W., Slavin, J. D., Svestka, J., Witt, A. N., and Zank, G. P. (1999). Dust in the Local Interstellar Wind. Astrophys. J., 525, 492-516.

Frisch, P. C., Grodnicki, L., and Welty, D. E. (2002). The Velocity Distribution of the Nearest Interstellar Gas. Astrophys. J., 574, 834-846.

Frisch, P. C., Bzowski, M., Grün, E., Izmodenov, V., Krüger, H., Linsky, J. L., McComas, D. J., Möbius, E., Redfield, S., Schwadron, N., Shelton, R. R., Slavin, J. D., and Wood, B. E. (2009). The Galactic Environment of the Sun: Interstellar Material Inside and Outside of the Heliosphere. Space Sci. Rev., pages 28-+.

Frisch, P. C., Andersson, B., Berdyugin, A., Funsten, H. O., Magalhaes, M., McComas, D. J., Piirola, V., Schwadron, N. A., Slavin, J. D., and Wiktorowicz, S. J. (2010). Comparisons of the Interstellar Magnetic Field Directions obtained from the IBEX Ribbon and Interstellar Polarizations. Astrophys. J., 724, 1473-1479.

Frisch, P. C., Redfield, S., and Slavin, J. D. (2011). The Interstellar Medium Surrounding the Sun. ARA\&A, submitted, 00, 00.

Gosling, J. T. (1964). A Study of the Relationship between Absorption-Time Profiles of PolarCap-Absorption Events and Forbush Decreases of Cosmic Ray Intensity. J. Geophys. Res., 69, 1233-1238.

Haud, U. (2010). Gaussian decomposition of HI surveys. V. Search for very cold clouds. Astron. Astrophys., 514, A27+.

Hebrard, G., Mallouris, C., Ferlet, R., Koester, D., Lemoine, M., Vidal-Madjar, A., and York, D. (1999). Ultraviolet observations of Sirius A and Sirius B with HST-GHRS. An interstellar cloud with a possible low deuterium abundance. Astron. Astrophys., 350, 643-658.

Heiles, C. (1998). Whence the Local Bubble, Gum, Orion? GSH 238+00+09, A nearby major superbubble toward Galactic longitude 238 degrees. Astrophys. J., 498, 689-703.

Holzer, T. E. (1989). Interaction between the solar wind and the interstellar medium. ARA $\& A$, 27, 199-234

Kirkby, J. and Carslaw, K. S. (2006). Variations of Galactic Cosmic Rays and the Earth's Climate. In Solar Journey: The Significance of Our Galactic Environment for the Heliosphere and Earth, page 000. Springer, Ed. P. C. Frisch.

Lallement, R., Ferlet, R., Lagrange, A. M., Lemoine, M., and Vidal-Madjar, A. (1995). Local cloud structure from HST-GHRS. Astron. Astrophys., 304, 461-474.

Leske, R. A., Cummings, A. C., Cohen, C. M. S., and et al. (2008). Changing anomalous cosmic ray oxygen radial intensity gradients between $1 \mathrm{AU}$ and Voyager with the return to solar minimum. In International Cosmic Ray Conference, volume 1 of International Cosmic Ray Conference, pages 807-810.

McCracken, K. G. (2004). Geomagnetic and atmospheric effects upon the cosmogenic Be10 observed in polar ice. J. Geophys. Res., 109, 4101-+.

Mewaldt, R. A., Cummings, A. C., and Stone, E. C. (1998). Anomalous Cosmic Ray: Interstellar Interlopers in the Heliosphere and Magnetosphere. In S. T. Suess \& B. T. Tsurutani, editor, From the Sun, Auroras, Magnetic Storms, Solar Flares, Cosmic Rays, pages 133+ .

Meyer, D. M., Lauroesch, J. T., Heiles, C., Peek, J. E. G., and Engelhorn, K. (2006). A Cold Nearby Cloud inside the Local Bubble. Astrophys. J. Lett., 650, L67-L70. 
Möbius, E., Bzowski, M., Chalov, S., Fahr, H., Gloeckler, G., Izmodenov, V., Kallenbach, R., Lallement, R., McMullin, D., Noda, H., Oka, M., Pauluhn, A., Raymond, J., Ruciński, D., Skoug, R., Terasawa, T., Thompson, W., Vallerga, J., von Steiger, R., and Witte, M. (2004). Synopsis of the interstellar He parameters from combined neutral gas, pickup ion and UV scattering observations and related consequences. Astron. Astrophys., 426, 897-907.

Müller, H., Woodman, L. M., and Zank, G. P. (2008). Heliospheric termination shock strength from a multi-fluid model. In G. Li, Q. Hu, O. Verkhoglyadova, G. P. Zank, R. P. Lin, \& J. Luhmann, editor, American Institute of Physics Conference Series, volume 1039 of American Institute of Physics Conference Series, pages 384-389.

Müller, H., Frisch, P. C., Fields, B. D., and Zank, G. P. (2009). The Heliosphere in Time. Space Sci. Rev., 143, 415-425.

Müller, H.-R., Frisch, P. C., Florinski, V., and Zank, G. P. (2006). Heliospheric Response to Different Possible Interstellar Environments. Astrophys. J., 647, 1491-1505.

Müller, H.-R., Frisch, P. C., Florinski, V., and Zank, G. P. (2006). Heliospheric response to different possible interstellar environments. Astrophys. J., 647, 1491-1505.

Muscheler, R., Beer, J., Kubik, P. W., and Synal, H. (2005). Geomagnetic field intensity during the last 60,000 years based on Be10 and Cl36 from the Summit ice cores and C14. Quaternary Science Reviews, 24, 1849-1860.

Perryman, M. A. C. (1997). The HIPPARCOS Catalogue. Astron. Astrophys., 323, L49-L52.

Pogorelov, N. V., Borovikov, S. N., Zank, G. P., and Ogino, T. (2009). Three-Dimensional Features of the Outer Heliosphere Due to Coupling Between the Interstellar and Interplanetary Magnetic Fields. III. The Effects of Solar Rotation and Activity Cycle. Astrophys. $J ., 696,1478-1490$.

Redfield, S. and Linsky, J. L. (2004a). The Structure of the Local Interstellar Medium. II. Observations of D I, C II, N I, O I, Al II, and Si II toward Stars within 100 Parsecs. Astrophys. J., 602, 776-802.

Redfield, S. and Linsky, J. L. (2004b). The Structure of the Local Interstellar Medium. III. Temperature and Turbulence. Astrophys. J., 613, 1004-1022.

Redfield, S. and Linsky, J. L. (2008). The Structure of the Local Interstellar Medium. IV. Dynamics, Morphology, Physical Properties, and Implications of Cloud-Cloud Interactions. Astrophys. J., 673, 283-314.

Salvati, M. (2010). The local Galactic magnetic field in the direction of Geminga. Astron. Astrophys., 513, A28+.

Schönrich, R., Binney, J., and Dehnen, W. (2010). Local kinematics and the local standard of rest. $M N R A S, \mathbf{4 0 3}, 1829-1833$.

Slavin, J. D. and Frisch, P. C. (2008). The boundary conditions of the heliosphere: photoionization models constrained by interstellar and in situ data. Astron. Astrophys., 491, 53-68.

Sonett, C. P., Morfill, G. E., and Jokipii, J. R. (1987). Interstellar Shock Waves and 10Be from Ice Cores. Nature, 330, 458.

Stanimirović, S. (2009). Exotic Clouds in the Local Interstellar Medium. Space Sci. Rev., 143, $291-301$.

Usoskin, I. G. (2008). A History of Solar Activity over Millennia. Living Reviews in Solar Physics, 5, 3-+.

Webber, W. R., Higbie, P. R., and McCracken, K. G. (2007). Production of the cosmogenic isotopes ${ }^{3} \mathrm{H},{ }^{7} \mathrm{Be},{ }^{10} \mathrm{Be}$, and ${ }^{36} \mathrm{Cl}$ in the Earth's atmosphere by solar and galactic cosmic rays. Journal of Geophysical Research (Space Physics), 112, 10106-+.

Wolff, B., Koester, D., and Lallement, R. (1999). Evidence for an Ionization Gradient in the Local Interstellar Medium: EUVE Observations of White Dwarfs. Astron. Astrophys., 346, 969-978.

Wolleben, M. (2007). A New Model for the Loop I (North Polar Spur) Region. Astrophys. J., 664, 349-356.

Wood, B. E., Redfield, S., Linsky, J. L., Müller, H.-R., and Zank, G. P. (2005). Stellar Ly $\alpha$ Emission Lines in the Hubble Space Telescope Archive: Intrinsic Line Fluxes and Absorption from the Heliosphere and Astrospheres. Astrophys. J. Suppl., 159, 118-140.

Yeghikyan, A. and Fahr, H. (2006). Accretion of interstellar material into the heliosphere and onto Earth, in Solar Journey: The Significance of Our Galactic Environment for the Heliosphere and Earth, Ed. P. C. Frisch. Springer.

Zank, G. P. and Müller, H.-R. (2003). The dynamical heliosphere. J. Geophys. Res., 108. 